\title{
Convolution neural network for the diagnosis of wireless capsule endoscopy: a systematic review and meta-analysis
}

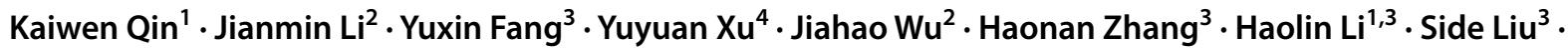 \\ Qingyuan $\mathrm{Li}^{3}$ (B)
}

Received: 5 May 2021 / Accepted: 7 August 2021 / Published online: 23 August 2021

(C) The Author(s) 2021

\begin{abstract}
Background Wireless capsule endoscopy (WCE) is considered to be a powerful instrument for the diagnosis of intestine diseases. Convolution neural network $(\mathrm{CNN})$ is a type of artificial intelligence that has the potential to assist the detection of WCE images. We aimed to perform a systematic review of the current research progress to the CNN application in WCE. Methods A search in PubMed, SinoMed, and Web of Science was conducted to collect all original publications about CNN implementation in WCE. Assessment of the risk of bias was performed by Quality Assessment of Diagnostic Accuracy Studies-2 risk list. Pooled sensitivity and specificity were calculated by an exact binominal rendition of the bivariate mixedeffects regression model. $I^{2}$ was used for the evaluation of heterogeneity.

Results 16 articles with 23 independent studies were included. CNN application to WCE was divided into detection on erosion/ulcer, gastrointestinal bleeding (GI bleeding), and polyps/cancer. The pooled sensitivity of CNN for erosion/ulcer is 0.96 [95\% CI 0.91, 0.98], for GI bleeding is 0.97 (95\% CI 0.93-0.99), and for polyps/cancer is 0.97 (95\% CI 0.82-0.99). The corresponding specificity of CNN for erosion/ulcer is 0.97 (95\% CI 0.93-0.99), for GI bleeding is 1.00 (95\% CI 0.99-1.00), and for polyps/cancer is 0.98 (95\% CI 0.92-0.99).

Conclusion Based on our meta-analysis, CNN-dependent diagnosis of erosion/ulcer, GI bleeding, and polyps/cancer approached a high-level performance because of its high sensitivity and specificity. Therefore, future perspective, CNN has the potential to become an important assistant for the diagnosis of WCE.
\end{abstract}

Keywords Deep learning · Convolutional neural network · Capsule endoscopy

Kaiwen Qin and Jianmin Li have contributed equally to this work.

Qingyuan Li

liqingyuan09@smu.edu.cn

1 Nanfang Hospital (The First School of Clinical Medicine), Southern Medical University, Guangzhou, Guangdong, China

2 Guangzhou SiDe MedTech Co.,Ltd, Guangzhou, Guangdong, China

3 Guangdong Provincial Key Laboratory of Gastroenterology, Department of Gastroenterology, Nanfang Hospital, Southern Medical University, No. 1838, Guangzhou Avenue North, Guangzhou, Guangdong, China

4 State Key Laboratory of Organ Failure Research, Guangdong Provincial Key Laboratory of Viral Hepatitis Research, Department of Hepatology Unit and Infectious Diseases, Nanfang Hospital, Southern Medical University, Guangzhou, Guangdong, China

\begin{tabular}{ll}
\multicolumn{2}{l}{ Abbreviations } \\
AI & Intelligence \\
CNN & Convolution neural network \\
FN & False negative \\
FP & False positive \\
GI & Gastrointestinal \\
QUADAS-2 & Quality assessment of diagnostic accuracy \\
& studies-2 \\
SROC & Summary receiver operating characteristic \\
TN & True negative \\
TP & True positive \\
WCE & Wireless capsule endoscopy
\end{tabular}

Wireless capsule endoscopy (WCE) is a powerful medical instrument for the screening and diagnosis of intestine diseases [1]. According to the clinical practice of ESGE, capsule endoscopy is the first-line investigation in patients with obscure gastrointestinal bleeding [2]. It is also an important 
tool for the surveillance of Crohn's disease, polyposis syndromes, and small-bowel cancers [1]. However, the defect of long reading time and the large number of frames restrict the development of WCE [3]. An average of 12,000 images are captured in a single WCE session, and gastroenterologists manually read WCE films with an average reading time of $30-40 \min [2,4]$.

"Deep learning" is an advanced form of machine learning, which is classified and fed back through multi-layer feature extraction [5]. The most popular learning algorithm for image analysis is the convolutional neural network (CNN) [6]. It can automatically and adaptively learn spatial hierarchies of features through backpropagation by using multiple building blocks [7]. Recently, CNN has attracted great attention and displays great performance in range of image recognition tasks including radiology [8], nephrology [9], and skin lesions [10]. More excitedly, CNN has been successfully used in many clinical applications such as the classification, detection, and segmentation tasks of images in radiology [11]. The diversity of machine learning methods is shown in Fig. 1.

In the field of endoscopy, the CNN model under supervised learning is the most widely used artificial intelligence (AI) method and is growing mature. The application of it can be divided into two groups: Computer-aided examination and diagnosis [12], which is widely used in the detection of various lesions such as gastric cancer, esophageal cancer, Intestine tumor, and Cokic Polyp, with the sensitivity and specificity which are much higher than experienced physicians [13-17].

Thus, deep learning has the potential to automatically detect diseases and shorten WCE reading time. In this study, we constructed a systematic review and meta-analysis to assess the application and performance of $\mathrm{CNN}$ for WCE.

\section{Materials and methods}

We conducted this review in accordance with the Preferred Reporting Items for systematic reviews and meta-analysis (PRISMA2020) [18]. The Grading of Recommendation Assessment, Development, and Evaluation (GRADE) is conducted for assessment of the quality of evidence [19]. The checklist of PRISMA2020 can be approached in Supplementary Table 1.

\section{Search strategy}

We systematically searched studies that assessed the accuracy of $\mathrm{CNN}$ for the diagnosis of gastrointestinal diseases by the use of WCE via PubMed, SinoMed, and Web of Science.

The search formula and corresponding search results of each database can be achieved in Supplementary Tables 2 and 3. We searched the databases between Jan 1, 2016, and March 15, 2021. We also searched the reference list of each primary study identified and previous systematic reviews.

\section{Study selection}

Two reviewers (KQ and JL) independently screened the titles and abstracts to determine whether the studies met inclusion criteria. Inclusion was based on titles and abstracts, as well as the full-text article.

Only those studies which are associated with both WCE and CNN can be selected. Primary studies that only use normal endoscopy or did not include the usage of CNN are excluded from our research.

Furthermore, the studies had to provide sufficient information to construct the $2 \times 2$ contingency table (true and false positives and negatives). Calculation methods of accuracy, specificity, and sensitivity are shown below.

Accuracy $=\frac{\mathrm{TP}+\mathrm{TN}}{\mathrm{TP}+\mathrm{TN}+\mathrm{FP}+\mathrm{FN}}$,

Sensitivity $=\frac{\mathrm{TP}}{\mathrm{TP}+\mathrm{FN}}$,

Specificity $=\frac{\mathrm{TN}}{\mathrm{TN}+\mathrm{FP}}$.

We only included publications written in English. Animal experiments, reviews, correspondences, case reports, expert opinions, and editorials were excluded. Disagreements in the inclusion process were resolved by a third reviewer.

\section{Quality assessment and risk of bias}

To evaluate the risk of bias, two reviewers independently applied the Quality Assessment of Diagnostic Accuracy Studies-2 (QUADAS-2) risk checklist [20] for the testing of bias risk of each study. Details of the list can be approached in Supplementary Table 4. Revman 5.4 (Cochrane Collaboration, London, United Kingdom) was used for the assessment of QUADAS-2 and bias risk.

\section{Data extraction}

Data from all included studies were collected into a standardized data extraction sheet, which included the year of publication, study design, application of pathology, type of database, algorithm, capsule brand, training set, validation set, and test set. The investigator also recorded the number of true and false positives and negatives. We contacted the corresponding authors if necessary information was needed. 
A

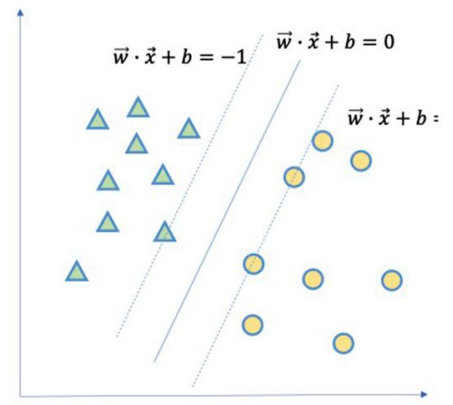

B

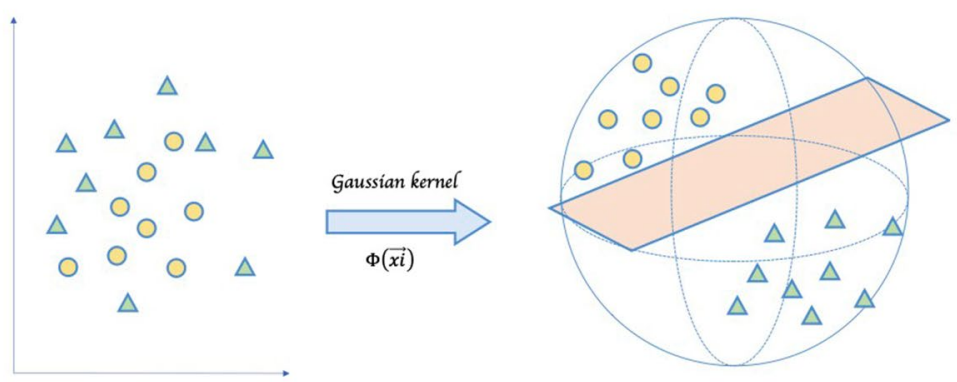

\section{C}

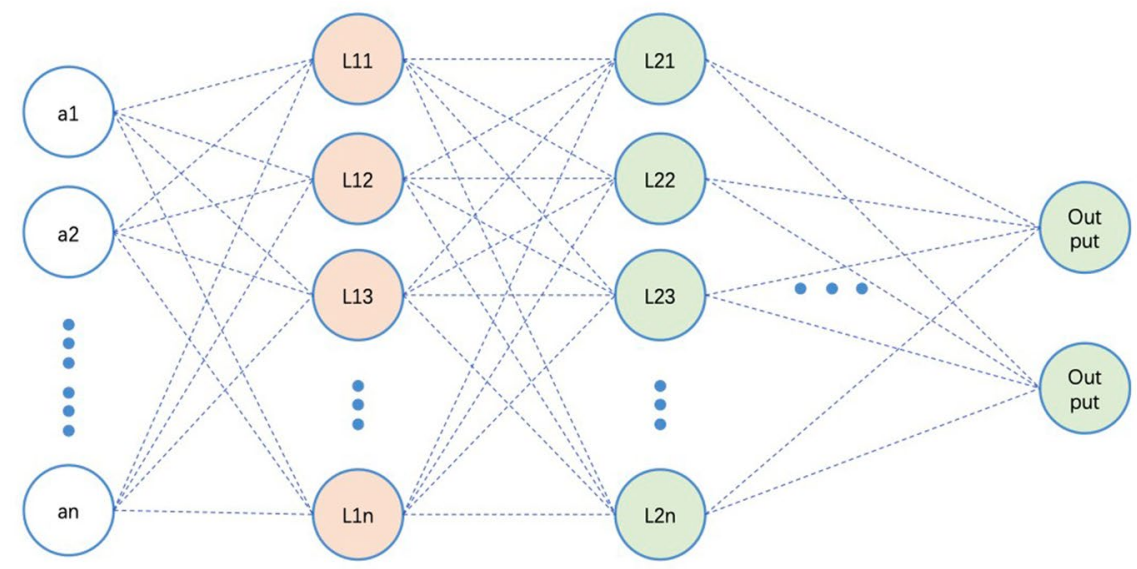

D
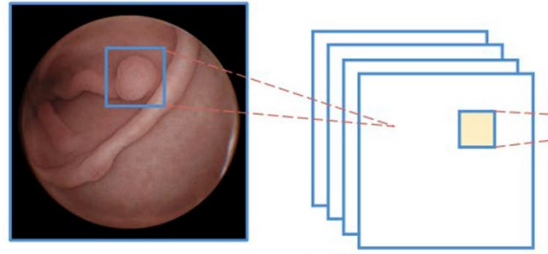

convolution

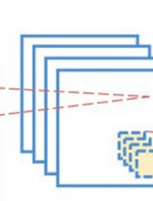

pooling

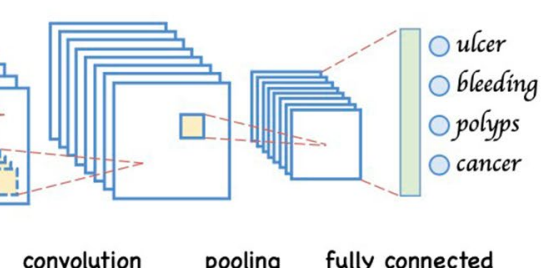

convolution pooling fully connected
Fig. 1 Different kind of machine learning algorithm. A Support vector machine (SVM) of linear classification. The data were classified with optimal hyper plane. B SVM of non-linear classification. When the data are linearly indivisible, a kernel function is used to map data to high dimensional space. $\mathbf{C}$ The structure of traditional neural network. An input is passed through the network layers using random weight, by the back propagation, the network fine-tunes the weights based on the error between the calculated output and the actual desired output. However, the large amount of linkage between different nodes of each layers greatly increases the number of parameters and the complexity of the algorithm. D Brief schematic diagram of $\mathrm{CNN}$
If a study provides multiple contingency tables related to diagnostic accuracy due to different algorithms, we assume that these contingency tables are independent of each other. We extract the contingency table with the highest overall accuracy into our study for analysis.

\section{Statistical analysis}

A subgroup analysis of the three most frequent types of lesions was performed to evaluate the quality of CNN in WCE image diagnosis, which are respectively erosion/ulcer, GI bleeding, and polyps/cancer. We tabulated true positives, false negatives, false positives, and true negatives of each 
research, which were used to calculate sensitivity and specificity and a corresponding CI.

To synthesis data, an exact binominal rendition of the bivariate mixed-effects regression model developed by van Houwelingen [21] was used for our analysis. This model does not transform pairs of sensitivity and specificity of individual studies into a single indicator of diagnostic accuracy, but preserves the two-dimensional nature of the data taking into account any correlation between the two [22]. The mean logit sensitivity and specificity with their standard error and 95\% CIs, the between-study variability in logit sensitivity and specificity, and covariance between them were estimated based on this model.

The original receiver operating curve scale was used to back-transform these quantities to obtain summary sensitivity, specificity, and diagnostic odds ratios. We then used the derived logit estimates of sensitivity, specificity, and respective variances to construct a hierarchical summary receiver operating curve for $\mathrm{CNN}$ with summary operating points for sensitivity and specificity on the curves and a 95\% confidence contour ellipsoid. Additionally, the Fagan nomogram was used for the diagnosis of CNN.

$I^{2}$ was used to assess heterogeneity, $I^{2}$ values $>50 \%$ were considered with significant heterogeneity. Study-level covariates can be used in meta-regression to combine results from multiple studies with attention to between-study variation [23]. To investigate publication bias, we constructed Deeks' funnel plots of asymmetry [24].

MIDAS module for STATA (version 15) was used for the meta-regression and bivariate summary receiver operating curve analysis. Graphs were produced with the MIDAS module and the QUADAS for Revman (version 5.4).

\section{Certainty assessment}

The five GRADE considerations (risk of bias, indirectness, inconsistency, precision, and publication bias) are used to assess the certainty of evidence in our research [19]. We assess our certainty of evidence as high, moderate, low, and very low. GRADE pro GDT software is used for the process of assessment and the preparation of the "Summary of finds" table. We justified all decisions to down- or up-grade the certainty of studies using footnotes.

\section{Results}

The database search of us retrieved 178 articles from PubMed, SinoMed, and Web of Science. First of all, 87 repeated articles were excluded. After title and abstract screening, 59 articles were excluded; finally, 16 articles were excluded after the full-text screening, leaving 16 articles with 23 independent studies for inclusion (when two or more lesions appear in one article, we regard them as independent studies.) (Fig. 2).

\section{Describe summary of the results}

In many studies, researchers reported the diagnostic accuracy of different diseases. Thus, we selected the three most representative lesions in the Department of Gastroenterology for subgroup analysis, which are separately ulcer/erosion (nine studies), GI bleeding (seven studies), and polyps/cancer (seven studies). [25-40]

In total, 69,991,447 WCE images in which there are 15,291,964 lesion WCE images were included. All of those studies are retrospective studies. 13 of them use private databases of images while 3 applied online databases.

Most of the studies used CNN for automatic detection, only one of them applied handwork and CNN joint applied. Pillcam and Ankon technology are the two widest used brands of WCE. All the research reported a relatively high accuracy, which is up to $80 \%$, while most of those studies' sensitivity and specificity are around $90 \%$.

For the detection of ulcers and erosions, Sen Wang et al. [31] used 49,064 images in which there are 24,839 lesion images. An accuracy of $92.1 \%$ was achieved. As for the diagnosis of GI bleeding, Aoki et al. [37] reported a 99.9\% accuracy with the usage of ResNet-50. In the determination of Polyps, Zhen Ding et al. [33] applied an extensive training set and validation set with 18,068,055 normal images and 5,912,433 polyps' images and achieve an accuracy of nearly $100 \%$.

The summary of studies that applied CNN techniques for WCE image analysis was listed in Table 1.

\section{Quality assessment}

According to the QUADAS-2 tool, eight of the 23 studies scored a high risk of bias in patient selection, because they did not clearly state the standard of the included images and patients, and we are not sure whether the patients' sample is a continuous cohort over a period of time. However, all of those studies scored a low risk in index test, reference standard, and flow and timing, which guarantees a low risk of bias. The summary of the quality assessment is presented in Fig. 3.

\section{Diagnostic accuracy analysis}

The detection of erosion/ulcer, GI bleeding, and polyps/cancer respectively contains nine, seven, and seven independent studies. Figure 4 shows the sensitivity and specificity of included studies. The pooled sensitivity of CNN for erosion/ ulcer is 0.96 (95\% CI 0.91, 0.98), for GI bleeding is 0.97 (95\% CI 0.93-0.99), and for polyps/cancer is 0.97 (95\% CI 


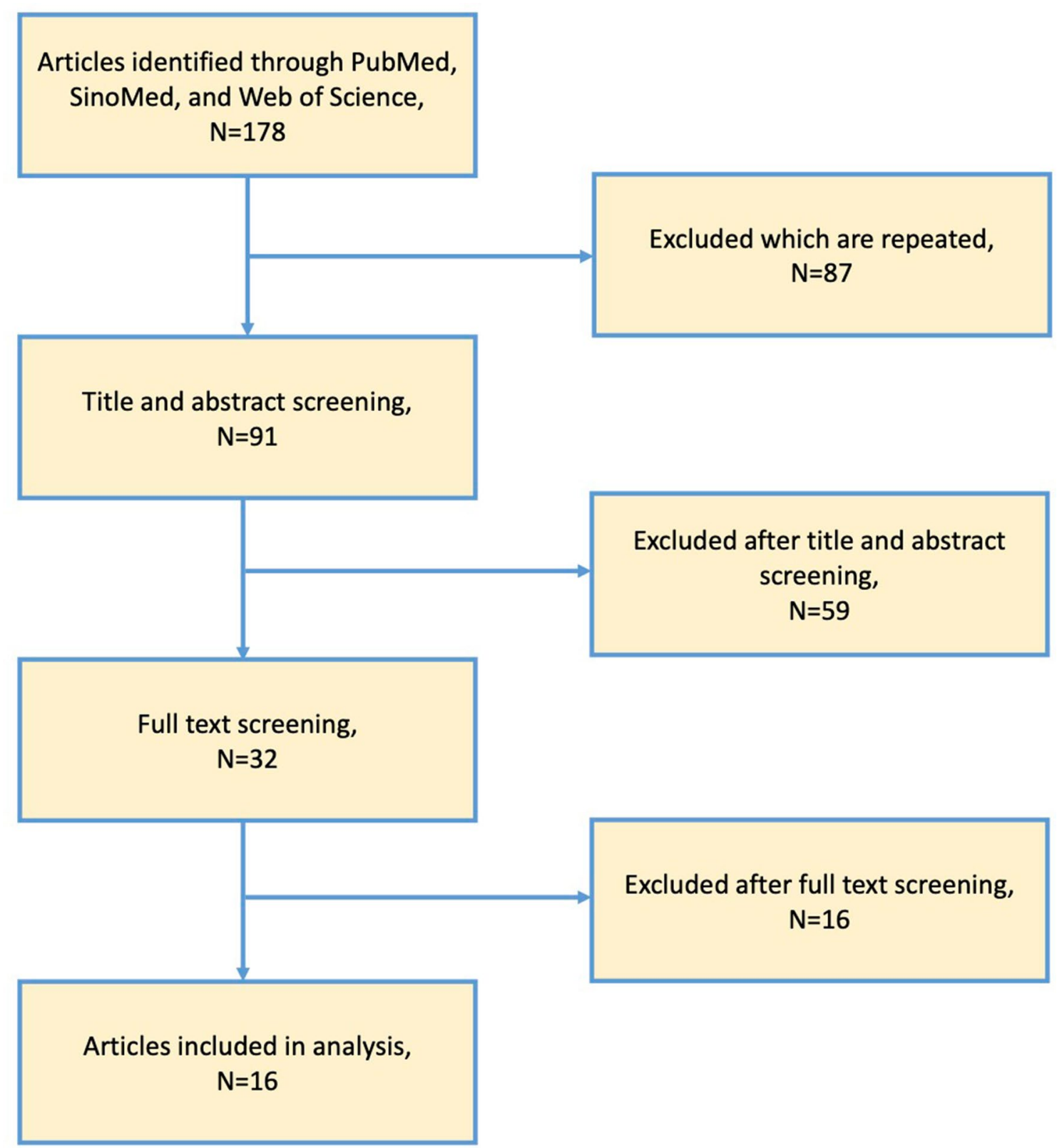

Fig. 2 Flow diagram of included studies search of using PubMed, SinoMed, and Web of Science

0.82-0.99). The corresponding specificity of CNN for erosion/ulcer is 0.97 (95\% CI 0.93-0.99), for GI bleeding is 1.00 (95\% CI 0.99-1.00), and for polyps/cancer is $0.98(95 \%$ CI 0.92-0.99). (Table 2).

The Fagan images used to describe the post-test probability are shown in Fig. 5. For erosion/ulcer, GI bleeding, and polyps/cancer, the Post-Prob-Pos (posterior probability positive) were separately $0.97,1.00$, and 0.98 while the PostProb-Neg (posterior probability negative) were respectively $0.04,0.03$, and 0.03 .
Supplementary Fig. 1 presents the bivariate summary receiver operating characteristic (SROC) curves. All subgroups analysis of GI diseases scored a high risk of bias $(p<0.1)$, which were showed in the Deeks' funnel plot asymmetry test in Supplementary Fig. 2. Substantial heterogeneity exists among the studies (overall $I^{2}$ for erosion/ ulcer $100 \%$, for GI bleeding $99 \%$, for polyps/cancer $100 \%$ ). Results summary of data analysis were displayed in Table 2.

"CNN dependent diagnosis of erosion/ulcer, GI bleeding, and polyps/cancer approached a high-level performance." 


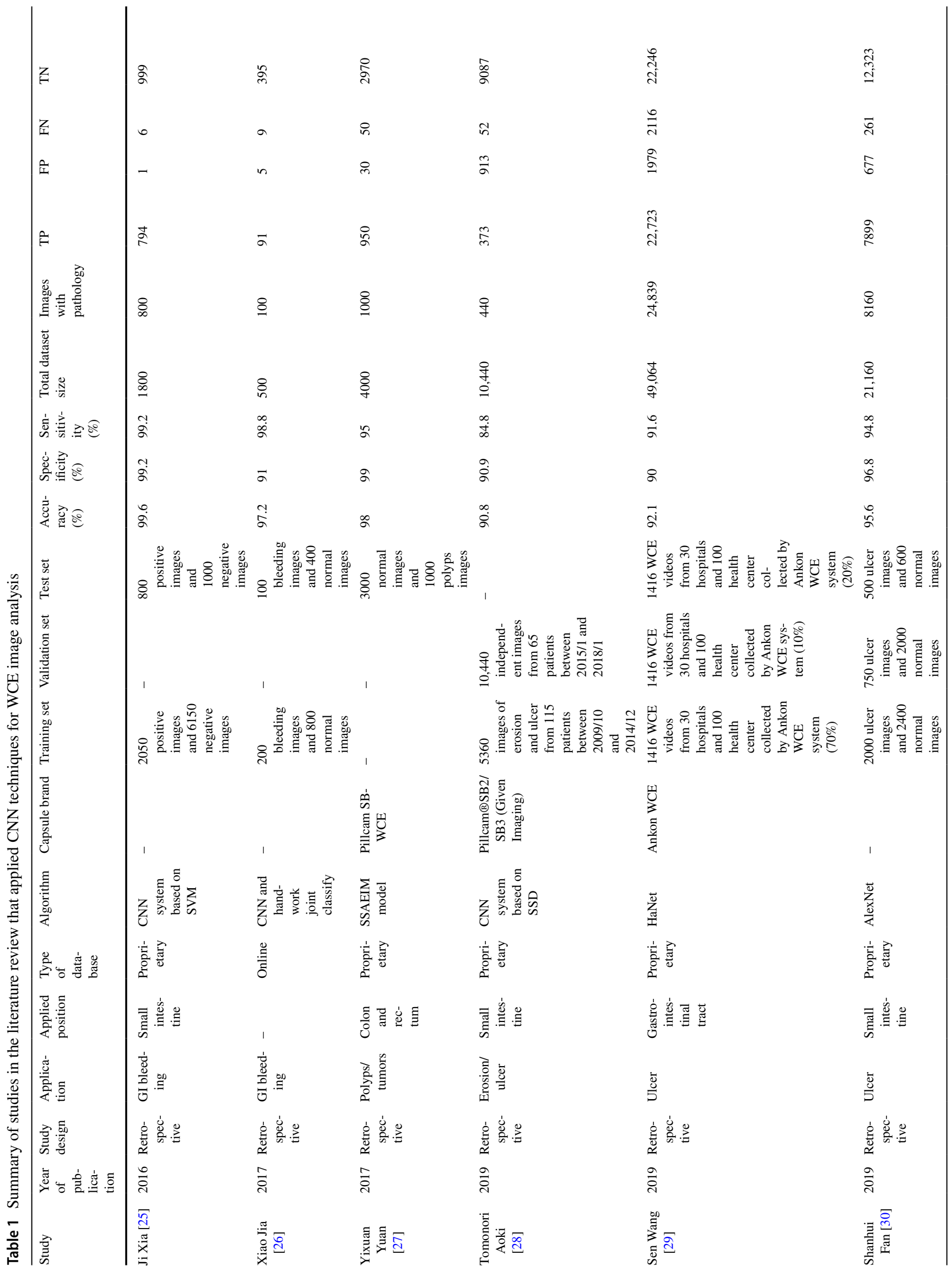




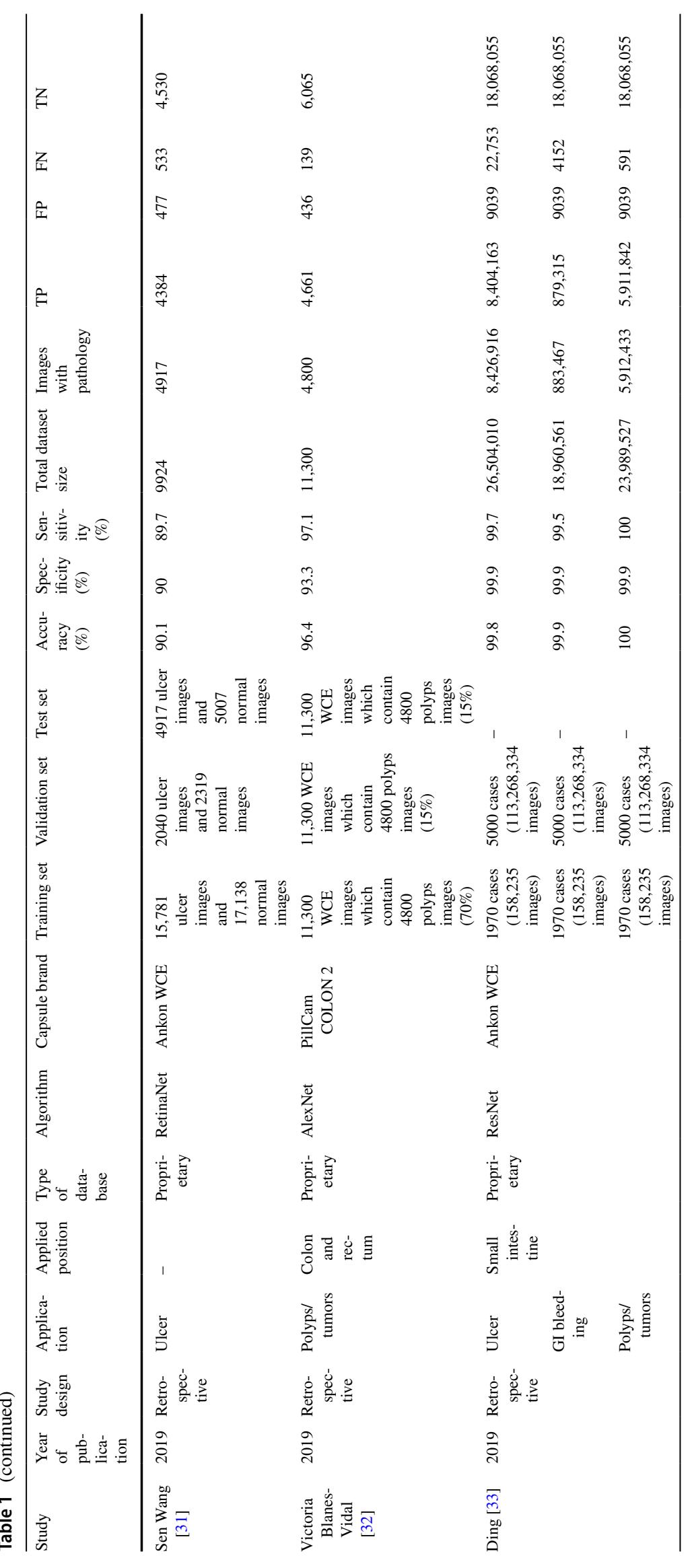




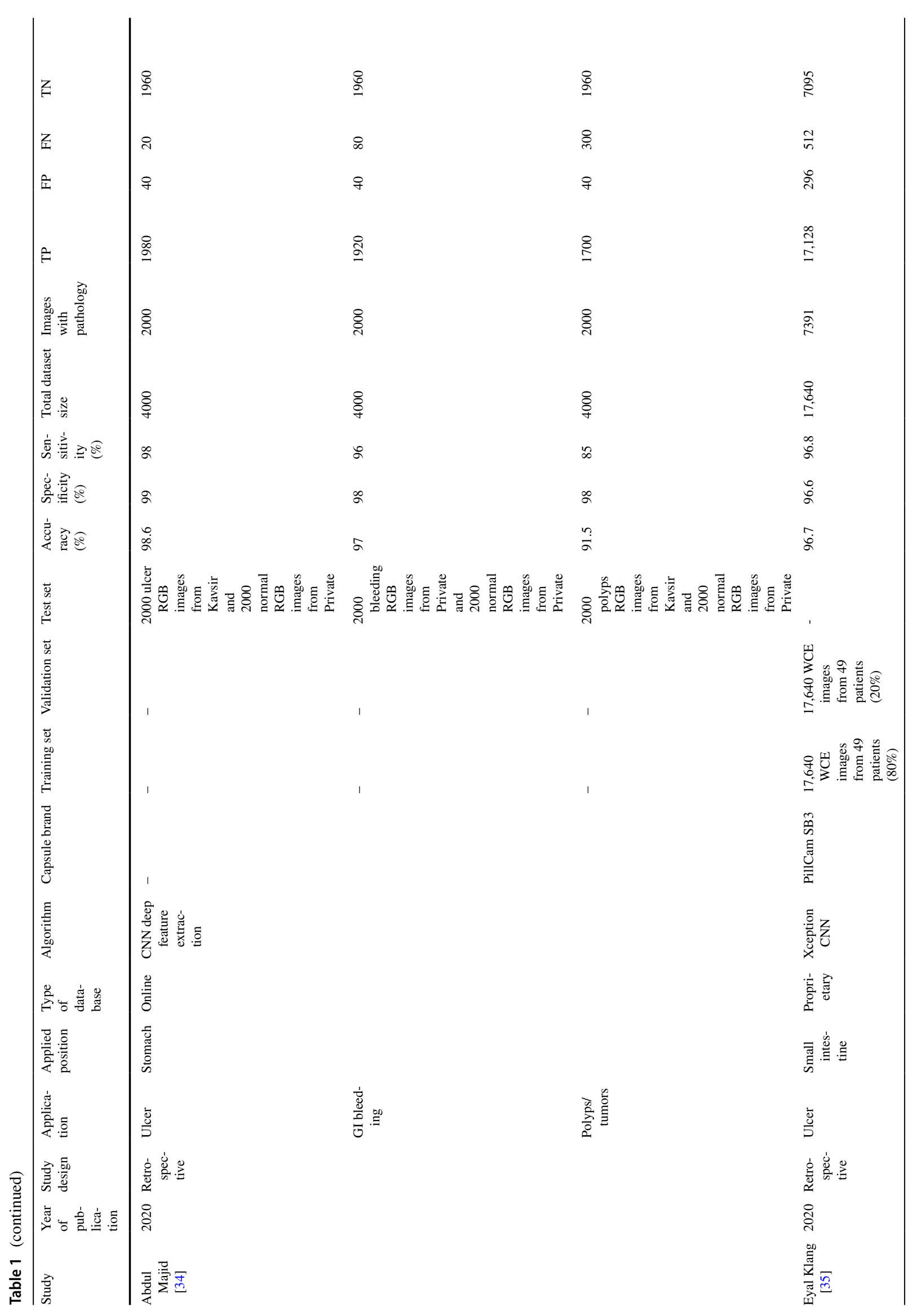




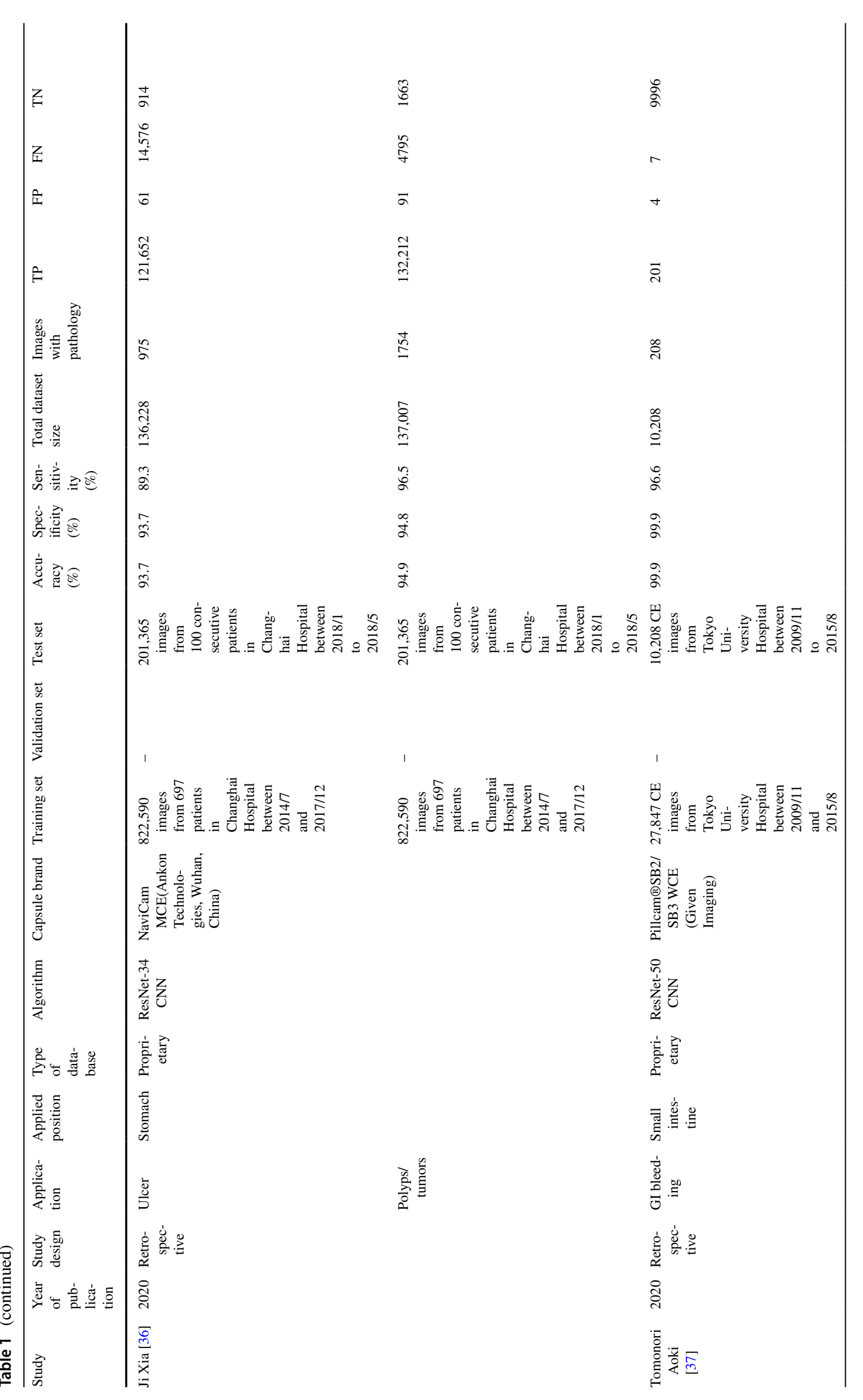




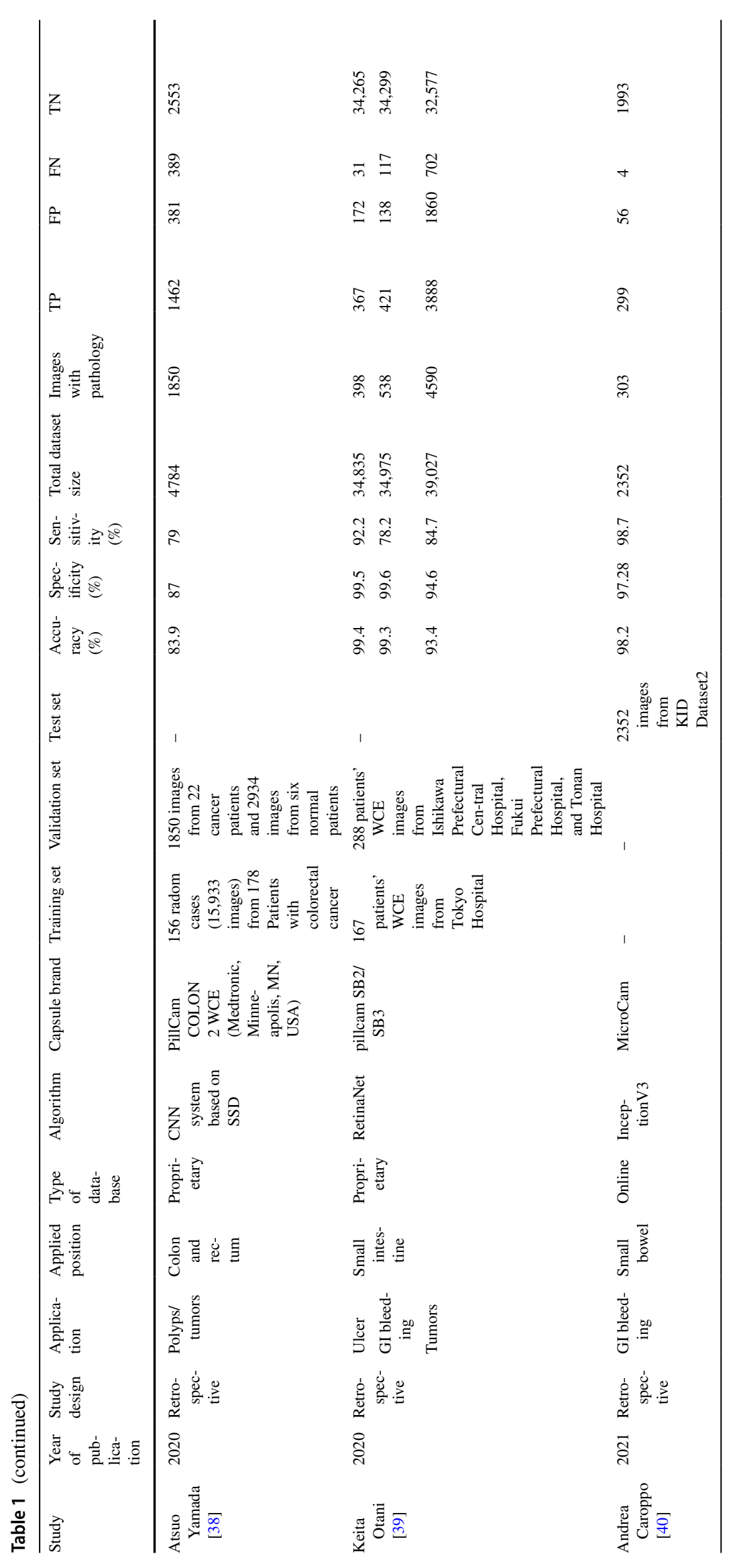


Fig. 3 Results of quality assessment. A Quality Assessment of Diagnostic Accuracy Studies-2 risk of bias assessment per clinical application. (Abdul Majid $2020 \mathrm{a} / \mathrm{b} / \mathrm{c}$ are respectively ulcer, bleeding and polyps. Ji Xia $2020 \mathrm{a} / \mathrm{b}$ are respectively ulcer and polyps. Ding 2019a/b/c are respectively ulcer, bleeding, and polyps. Keita Otani 2020a/b/c are respectively ulcer, bleeding, and tumors. Sen Wang 2019a/b are from two different articles, which is cited in Table 1.) $\mathbf{B}$ Risk of bias and applicability concerns graph: review authors' judgements about each domain presented as percentages across included studies
A

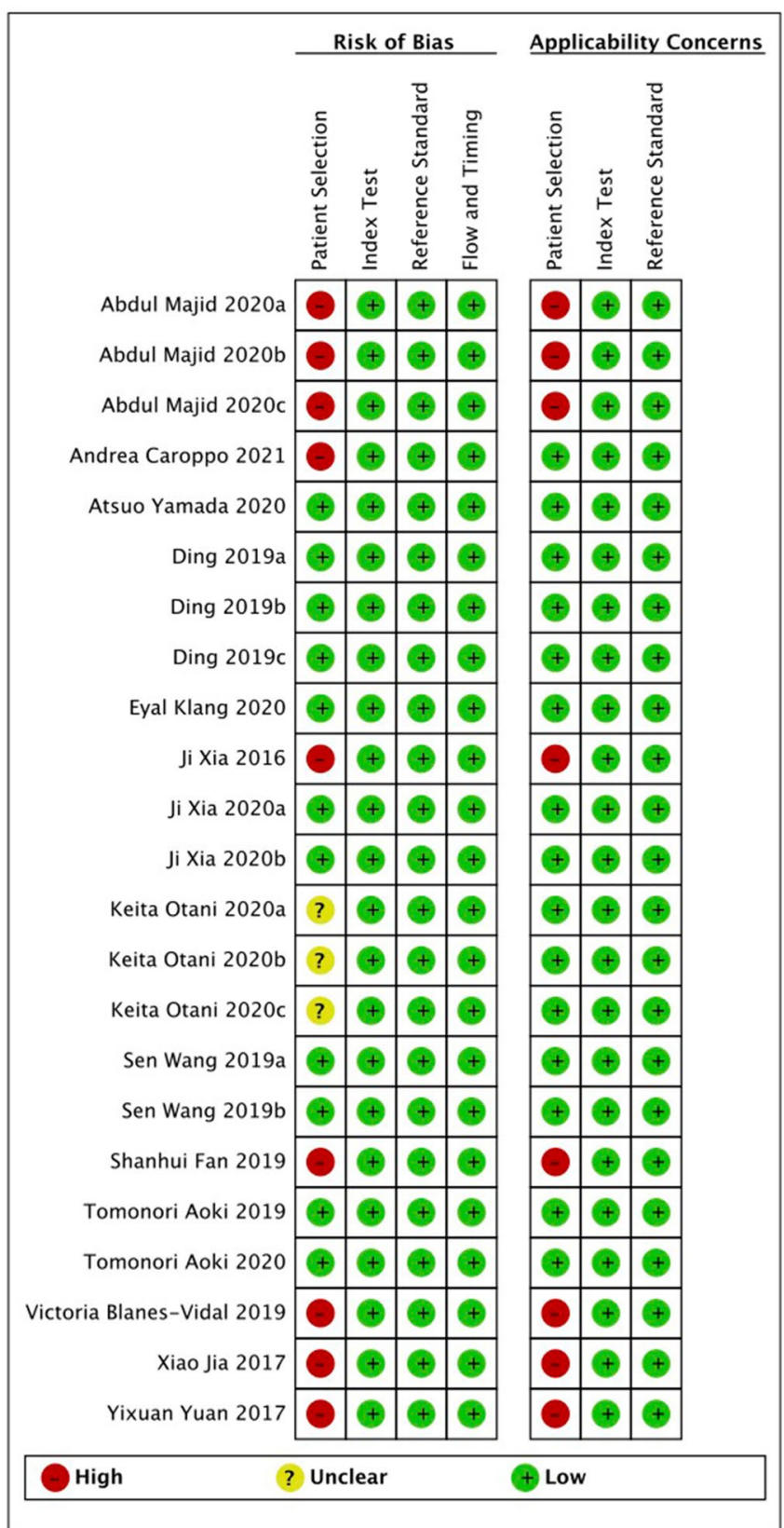

B

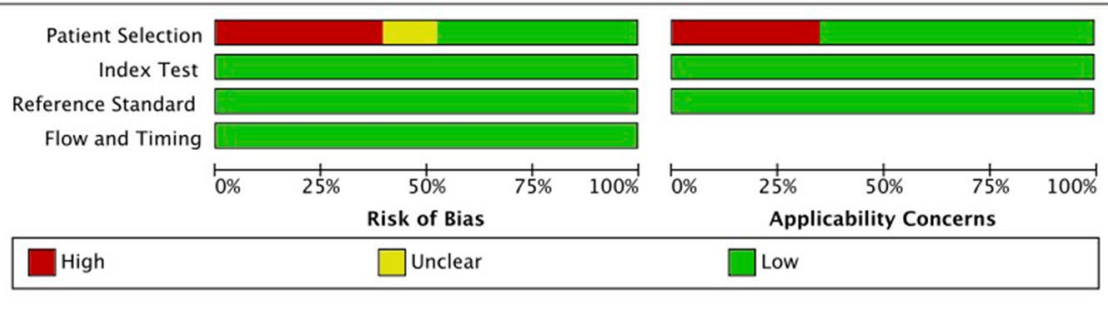


A

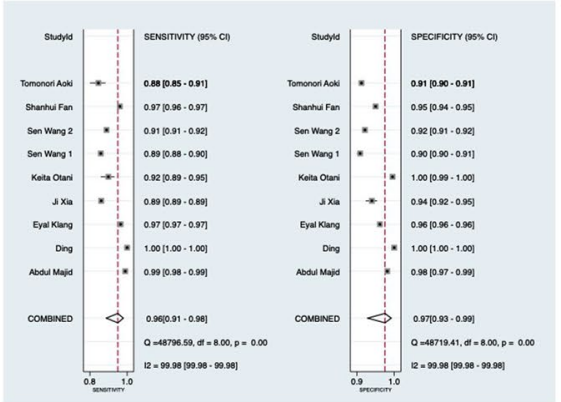

B

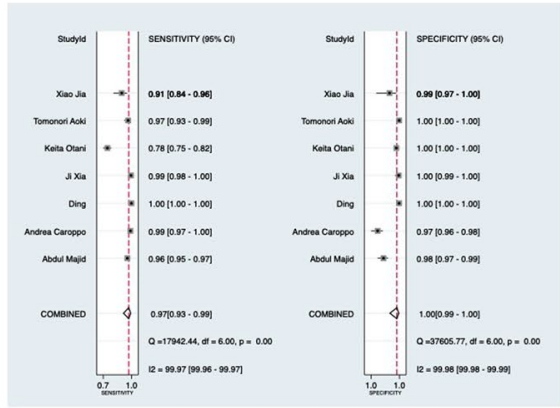

C

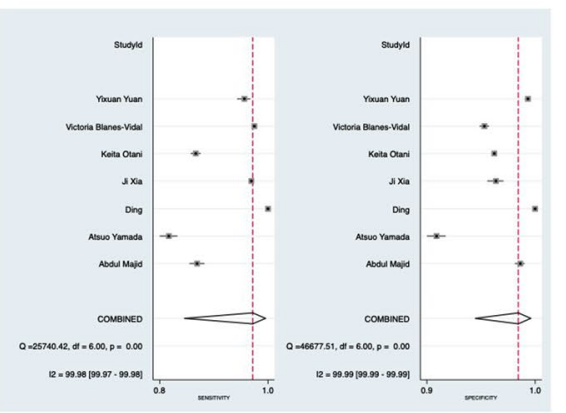

Fig. 4 Sensitivity and specificity of included studies as well as their corresponding results. A Detection of erosion or ulcer. B Detection of GI bleeding. C Detection of polyps or cancer

Table 2 Results summary of data analysis

\begin{tabular}{|c|c|c|c|c|c|c|c|c|}
\hline & $\begin{array}{l}\text { No. of } \\
\text { research }\end{array}$ & Sensitivity & Specificity & PLR & NLR & DOR & ROC area & $I$-square \\
\hline Erosion/ulcer & 9 & $\begin{array}{c}0.96[0.91, \\
0.98]\end{array}$ & $\begin{array}{c}0.97[0.93, \\
0.99]\end{array}$ & $\begin{array}{c}36.8[12.3, \\
110.1]\end{array}$ & $0.04[0.02,0.09]$ & $893[103,5834]$ & $\begin{array}{l}0.99[0.98- \\
1.00]\end{array}$ & $\begin{array}{r}100,95 \% \text { CI } \\
{[100-100]}\end{array}$ \\
\hline GI bleeding & 7 & $\begin{array}{c}0.97[0.93, \\
0.99]\end{array}$ & $\begin{array}{c}1.00[0.99, \\
1.00]\end{array}$ & $\begin{array}{c}289.4 \\
{[80.3,} \\
1043.0]\end{array}$ & $0.03[0.01,0.08]$ & $\begin{array}{l}10,291 \\
\quad[1539,68791]\end{array}$ & $\begin{array}{l}1.00[0.99- \\
1.00]\end{array}$ & $\begin{array}{r}99,95 \% \text { CI } \\
{[99-100]}\end{array}$ \\
\hline Polyps/tumors & 7 & $\begin{array}{c}0.97[0.82, \\
0.99]\end{array}$ & $\begin{array}{l}0.98[0.92, \\
0.99]\end{array}$ & $\begin{array}{c}42.7[11.3, \\
161.8]\end{array}$ & $0.03[0.01,0.21]$ & $1291[60,27808]$ & $\begin{array}{l}0.99[0.98- \\
1.00]\end{array}$ & $\begin{array}{r}100,95 \% \mathrm{CI} \\
{[100-100]}\end{array}$ \\
\hline
\end{tabular}

$P L R$ positive likelihood ratio, NLR negative likelihood ratio, DOR diagnostic odds ratio

A

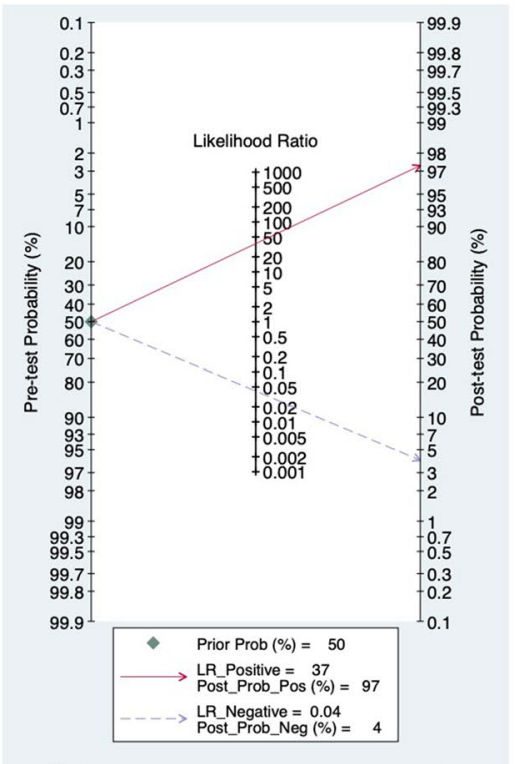

B

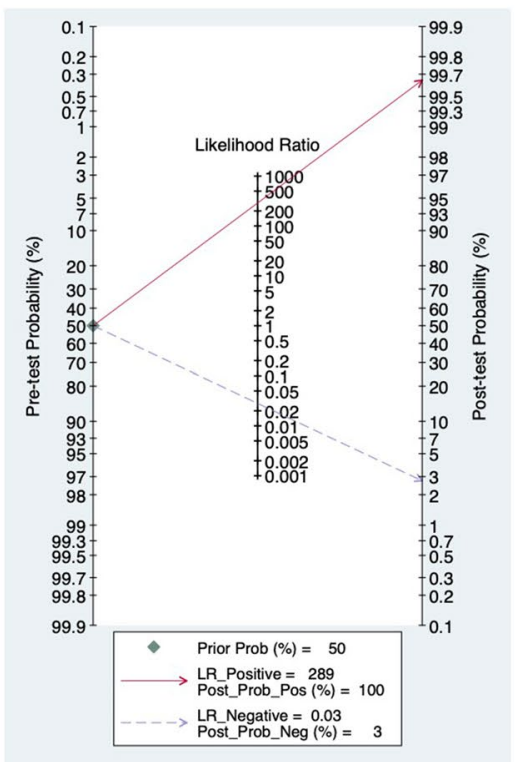

C

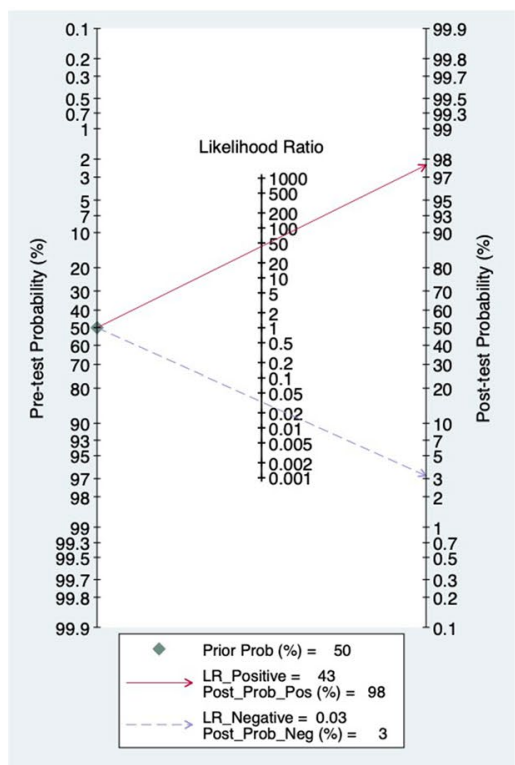

Fig. 5 Fagan image of post-test-probability. A Erosion or ulcer. B GI bleeding. C Polyps or cancer

This evidence downgrades two steps, once for inconsistency and once for publication bias, which results in low test-accuracy in five GRADE considerations. Summary of finds table of each subgroup analysis can be approached 
separately in Supplementary Tables 5, 6, and 7. The decisions to down- or up-grade the certainty were put in footnotes.

\section{Discussion}

Wireless capsule endoscopy is a technique widely used in the diagnosis of small intestinal diseases [41]. It has been used to detect small intestinal lesions that cannot be reached by traditional endoscopy. At the same time, although it is not the mainstream diagnostic method, capsule endoscopy is also used to explore some esophageal, gastric, and colorectal diseases [42]. Although WCE is convenient and painless, the high rate of miss diagnosis has always been its disadvantage, which is mostly related to people's limited reading ability and energy [43]. Due to the extension of film reading time, the readers' attention cannot be focused for a long time, further increasing the misdiagnosis rate [44]. With the development of CNN and the increasing accessibility of public databases, the application of AI in capsule endoscopy has been greatly developed, which reduces the burden of human readers [43].

AI algorithms, especially deep learning algorithms, have made significant progress in image recognition [45]. In the past few years, deep learning has totally changed the field of computer image processing and recognition [11]. Methods from convolutional neural network to variational autocoding have been widely used in the field of medical image analysis, which promotes the rapid development of medical image analysis [8], and it has been widely accepted that CNN has the power to contribute to the detection of various gastrointestinal diseases including erosion, ulcer, bleeding, polyps, and cancer [46].

So far, the application of CNN in the image diagnosis of WCE has attracted wide attention among the community of endoscopy. An increasing number of CNN research on WCE have been published. Mohan et al. [47] are the first team to conduct a systematic review on the CNN usage in WCE and reported a pooled accuracy of $95.4 \%$ on the diagnosis of GI ulcer and hemorrhage. Shelly Soffer et al. [48] also performed a meta-analysis on the CNN detection of ulcer and bleeding. Based on their work, we reviewed the application of CNN in WCE more comprehensively by incorporating more updated studies and included the diagnosis of polyps/ cancer lesions.

In our reviews of all studies, we found that $\mathrm{CNN}$ acted an excellent ability to the WCE diagnosis of the digestive tract. Almost every research included in our review shows an accuracy of more than $90 \%$, which is comparable with an experienced and senior endoscopist. Besides, high pooled sensitivity and specificity can also be achieved in the diagnosis of ulcer, bleeding, polyps, and cancer. This can indicate the value of clinical practice and reduce the massive and repetitive WCE images needed to be evaluated by human readers $[2,49]$. Some research included in our review also compared the performance of $\mathrm{CNN}$ with traditional machine learning methods to find the results that $\mathrm{CNN}$ was much better than others. For example, Fan's research indicates that the accuracy of CNN is nearly $25 \%$ higher than histogram-SVM for the diagnosis of ulcers [30]. Aoki et al. also report a 20\% higher sensitivity on CNN rather than SBI (a conventional tool used to automatically tag images depicting possible bleeding in the reading system) for the detection of bleeding [37]. The gap between CNN and traditional machine learning can be explained by their differences: the diagnosis of machine learning is based on man-selected characteristics such as color, shape, and pattern, which is the simulation of physicians. On the contrary, $\mathrm{CNN}$ is a learning system that can automatically extract the features through the training set [50]. However, because of the automatic process of detection, it's hard for us to find out how the network is constructed. Thus, CNN is often called the "black box" [11].

Besides image detection, $\mathrm{CNN}$ also shows great abilities to solve some shortcomings of WCE compared with normal endoscopy. Firstly, before the gastrointestinal examination of capsule endoscopy, diarrhea, and fasting are necessary to clean the gastrointestinal tract. However, it is still difficult to avoid the presence of intestinal contents (such as bile, bubbles, and food residues), which will hinder the observation of mucosa and affect the correct diagnosis and analysis of WCE [51]. Reinier Noorda et al. adopted an automatic evaluation system of capsule endoscopy cleanliness based on a new $\mathrm{CNN}$ architecture. By dividing the gastrointestinal tract cleanliness into four grades (poor, general, good, and excellent), the objective and automatic cleanliness evaluation were realized, and a good classification accuracy (95.23\%) was achieved [52]. On the field of cleanliness assessment of small-bowel capsule endoscopy, Romain Leenhardt et al. also reported an accuracy of $89.7 \%$ to determine whether the bowel preparation is enough or not [53]. Another major problem of capsule endoscopy is the retention at the gastroduodenal junction. Physicians often need to spend several hours observing whether the capsule has entered the duodenum or not [54]. Tao Gan et al. tested a CNN system for automatic detection of capsule endoscopy passing through the gastroduodenal junction, and the probability of judgment time error within 8 min reached $95.7 \%$, which indicate the ability of CNN to help endoscopes automatically determine gastric retention and reduce the time consuming and laborious work [55].

At present, the research of CNN in capsule gastroscopy diagnosis is still limited in the clinical research stage. All the studies are retrospective and most of them only focus on one or two kinds of diseases rather than comprehensive diagnosis. In addition, most of the research data are from 
single-center, and the clinical research based on multi-center has not been published yet, which will become the direction of our future efforts.

There are some limitations in our study: Firstly, the risk of bias for erosion/ulcers and polyps/cancer is relatively high. It is possibly because the number of cases of lesions like polyps or ulcer is less than that of GI bleeding, while their diagnosis of $\mathrm{AI}$ is much more complex, which results in fewer samples of erosion/ulcers, polyps/cancer, and leads to the differences in the data sets used for training and validation in different centers. Secondly, high heterogeneity exists among the studies included in this review, which may be due to the differences of algorithms in some studies, as well as the distinct strictness of experts in different centers for positive judgment of lesions. These limitations result in the low test-accuracy in the certainty assessment of GRADE consideration and may harm the accuracy and applicability of this review.

\section{Conclusion}

In conclusion, our research indicates that $\mathrm{CNN}$ has a considerable performance in WCE image diagnosis, and its quality in accuracy, sensitivity, and specificity has reached a relatively high level. With the development of algorithms and computer hardware, the accuracy of CNN will grow higher, and it will become an important tool to help doctors diagnose and play an irreplaceable role in future clinical applications. Besides, research on big data and multi-center will also be the trend of the process of AI application on WCE. Much more data and samples from various patients as training sets are more likely to improve the accuracy and reduce the risk of bias, to achieve the necessary conditions for this technology to be used in routine clinical practice.

Supplementary Information The online version contains supplementary material available at https://doi.org/10.1007/s00464-021-08689-3.

Acknowledgements This work was supported by the National Natural Science Funds of China (12026605), Guangdong Basic and Applied Basic Research Fund (2020A1515110916, 2021A1515010992), the Guangdong Medical Science and Technology Research Fund Project (A2020143), the College Students' Innovative Entrepreneurial Training Plan Program (202012121035X), the President Foundation of Nanfang Hospital, Southern Medical University (2018C027), and the Guangdong Science and Technology Plan Project (2017B020209003). We would like to acknowledge Pazhou Lab, Guangzhou for its support of this research.

Author contributions Q-YL and S-DL conceived and designed the study. K-WQ and Q-YL co-drafted of the manuscript. K-WQ and J-ML Searched the literature and do the assessment. Y-XF and Y-YX interpreted the data. J-HW, H-NZ, and H-LL critically reviewed the manuscript and offered proposals. All authors have read and proved the final manuscript.

\section{Declarations}

Disclosures Drs. Kaiwen Qin, Jianmin Li, Yuxin Fang, Yuyuan Xu, Jiahao Wu, Haonan Zhang, Haolin Li, Side Liu, and Qingyuan Li have no conflict of interest or financial ties to disclose.

Open Access This article is licensed under a Creative Commons Attribution 4.0 International License, which permits use, sharing, adaptation, distribution and reproduction in any medium or format, as long as you give appropriate credit to the original author(s) and the source, provide a link to the Creative Commons licence, and indicate if changes were made. The images or other third party material in this article are included in the article's Creative Commons licence, unless indicated otherwise in a credit line to the material. If material is not included in the article's Creative Commons licence and your intended use is not permitted by statutory regulation or exceeds the permitted use, you will need to obtain permission directly from the copyright holder. To view a copy of this licence, visit http://creativecommons.org/licenses/by/4.0/.

\section{References}

1. Enns RA, Hookey L, Armstrong D, Bernstein CN, Heitman SJ, Teshima C, Leontiadis GI, Tse F, Sadowski D (2017) Clinical practice guidelines for the use of video capsule endoscopy. Gastroenterology 152:497-514

2. Pennazio M, Spada C, Eliakim R, Keuchel M, May A, Mulder CJ, Rondonotti E, Adler SN, Albert J, Baltes P, Barbaro F, Cellier C, Charton JP, Delvaux M, Despott EJ, Domagk D, Klein A, McAlindon M, Rosa B, Rowse G, Sanders DS, Saurin JC, Sidhu R, Dumonceau JM, Hassan C, Gralnek IM (2015) Small-bowel capsule endoscopy and device-assisted enteroscopy for diagnosis and treatment of small-bowel disorders: European Society of Gastrointestinal Endoscopy (ESGE) Clinical Guideline. Endoscopy 47:352-376

3. Mishkin DS, Chuttani R, Croffie J, Disario J, Liu J, Shah R, Somogyi L, Tierney W, Song LM, Petersen BT (2006) ASGE technology status evaluation report: wireless capsule endoscopy. Gastrointest Endosc 63:539-545

4. Koulaouzidis A, Iakovidis DK, Karargyris A, Plevris JN (2015) Optimizing lesion detection in small-bowel capsule endoscopy: from present problems to future solutions. Expert Rev Gastroenterol Hepatol 9:217-235

5. Goodfellow I, Bengio Y, Courville A (2016) Deep learning. MIT press, Cambridge

6. Erickson BJ, Korfiatis P, Akkus Z, Kline T, Philbrick K (2017) Toolkits and libraries for deep learning. J Digit Imaging 30:400-405

7. Yamashita R, Nishio M, Do RKG, Togashi K (2018) Convolutional neural networks: an overview and application in radiology. Insights Imaging 9:611-629

8. Hosny A, Parmar C, Quackenbush J, Schwartz LH, Aerts H (2018) Artificial intelligence in radiology. Nat Rev Cancer 18:500-510

9. Niel O, Bastard P (2019) Artificial intelligence in nephrology: core concepts, clinical applications, and perspectives. Am J Kidney Dis 74:803-810

10. Aractingi S, Pellacani G (2019) Computational neural network in melanocytic lesions diagnosis: artificial intelligence to improve diagnosis in dermatology? Eur J Dermatol 29:4-7 
11. Chartrand G, Cheng PM, Vorontsov E, Drozdzal M, Turcotte S, Pal CJ, Kadoury S, Tang A (2017) Deep learning: a primer for radiologists. Radiographics 37:2113-2131

12. Chahal D, Byrne MF (2020) A primer on artificial intelligence and its application to endoscopy. Gastrointest Endosc 92:813-820. e814

13. Choi JY, Lee BS (2019) Ensemble of deep convolutional neural networks with Gabor face representations for face recognition. IEEE Trans Image Process. https://doi.org/10.1109/TIP.2019. 2958404

14. Ebigbo A, Mendel R, Probst A, Manzeneder J, Souza LA Jr, Papa JP, Palm C, Messmann H (2019) Computer-aided diagnosis using deep learning in the evaluation of early oesophageal adenocarcinoma. Gut 68:1143-1145

15. Hirasawa T, Aoyama K, Tanimoto T, Ishihara S, Shichijo S, Ozawa T, Ohnishi T, Fujishiro M, Matsuo K, Fujisaki J, Tada T (2018) Application of artificial intelligence using a convolutional neural network for detecting gastric cancer in endoscopic images. Gastric Cancer 21:653-660

16. Urban G, Tripathi P, Alkayali T, Mittal M, Jalali F, Karnes W, Baldi P (2018) Deep learning localizes and identifies polyps in real time with $96 \%$ accuracy in screening colonoscopy. Gastroenterology 155:1069-1078.e1068

17. Doherty GA, Moss AC, Cheifetz AS (2011) Capsule endoscopy for small-bowel evaluation in Crohn's disease. Gastrointest Endosc 74:167-175

18. Page MJ, Moher D, Bossuyt PM, Boutron I, Hoffmann TC, Mulrow CD, Shamseer L, Tetzlaff JM, Akl EA, Brennan SE, Chou R, Glanville J, Grimshaw JM, Hróbjartsson A, Lalu MM, Li T, Loder EW, Mayo-Wilson E, McDonald S, McGuinness LA, Stewart LA, Thomas J, Tricco AC, Welch VA, Whiting P, McKenzie JE (2021) PRISMA 2020 explanation and elaboration: updated guidance and exemplars for reporting systematic reviews. BMJ 372:n160

19. Guyatt GH, Oxman AD, Vist GE, Kunz R, Falck-Ytter Y, AlonsoCoello P, Schünemann HJ (2008) GRADE: an emerging consensus on rating quality of evidence and strength of recommendations. BMJ 336:924-926

20. Whiting P, Rutjes AW, Reitsma JB, Bossuyt PM, Kleijnen J (2003) The development of QUADAS: a tool for the quality assessment of studies of diagnostic accuracy included in systematic reviews. BMC Med Res Methodol 3:25

21. Van Houwelingen HC, Zwinderman KH, Stijnen T (1993) A bivariate approach to meta-analysis. Stat Med 12:2273-2284

22. Chu H, Cole SR (2006) Bivariate meta-analysis of sensitivity and specificity with sparse data: a generalized linear mixed model approach. J Clin Epidemiol 59:1331-1332 (author reply 1332-1333)

23. Ioannidis JP, Patsopoulos NA, Evangelou E (2007) Uncertainty in heterogeneity estimates in meta-analyses. BMJ 335:914-916

24. Deeks JJ, Macaskill P, Irwig L (2005) The performance of tests of publication bias and other sample size effects in systematic reviews of diagnostic test accuracy was assessed. J Clin Epidemiol 58:882-893

25. Xiao J, Meng MQ (2016) A deep convolutional neural network for bleeding detection in Wireless Capsule Endoscopy images. Annu Int Conf IEEE Eng Med Biol Soc 2016:639-642

26. Xiao J, Meng MQ (2017) Gastrointestinal bleeding detection in wireless capsule endoscopy images using handcrafted and CNN features. Annu Int Conf IEEE Eng Med Biol Soc 2017:3154-3157

27. Yuan Y, Meng MQ (2017) Deep learning for polyp recognition in wireless capsule endoscopy images. Med Phys 44:1379-1389

28. Aoki T, Yamada A, Aoyama K, Saito H, Tsuboi A, Nakada A, Niikura R, Fujishiro M, Oka S, Ishihara S, Matsuda T, Tanaka S, Koike K, Tada T (2019) Automatic detection of erosions and ulcerations in wireless capsule endoscopy images based on a deep convolutional neural network. Gastrointest Endosc 89:357-363. e352

29. Wang S, Xing Y, Zhang L, Gao H, Zhang H (2019) Deep Convolutional Neural Network for Ulcer Recognition in Wireless Capsule Endoscopy: Experimental Feasibility and Optimization. Comput Math Methods Med 2019:7546215

30. Fan S, Xu L, Fan Y, Wei K, Li L (2018) Computer-aided detection of small intestinal ulcer and erosion in wireless capsule endoscopy images. Phys Med Biol 63:165001

31. Wang S, Xing Y, Zhang L, Gao H, Zhang H (2019) A systematic evaluation and optimization of automatic detection of ulcers in wireless capsule endoscopy on a large dataset using deep convolutional neural networks. Phys Med Biol 64:235014

32. Blanes-Vidal V, Baatrup G, Nadimi ES (2019) Addressing priority challenges in the detection and assessment of colorectal polyps from capsule endoscopy and colonoscopy in colorectal cancer screening using machine learning. Acta Oncol 58:S29-S36

33. Ding Z, Shi H, Zhang H, Meng L, Fan M, Han C, Zhang K, Ming F, Xie X, Liu H, Liu J, Lin R, Hou X (2019) Gastroenterologistlevel identification of small-bowel diseases and normal variants by capsule endoscopy using a deep-learning model. Gastroenterology 157:1044-1054.e1045

34. Majid A, Khan MA, Yasmin M, Rehman A, Yousafzai A, Tariq U (2020) Classification of stomach infections: A paradigm of convolutional neural network along with classical features fusion and selection. Microsc Res Tech 83:562-576

35. Klang E, Barash Y, Margalit RY, Soffer S, Shimon O, Albshesh A, Ben-Horin S, Amitai MM, Eliakim R, Kopylov U (2020) Deep learning algorithms for automated detection of Crohn's disease ulcers by video capsule endoscopy. Gastrointest Endosc 91:606613.e602.

36. Xia J, Xia T, Pan J, Gao F, Wang S, Qian YY, Wang H, Zhao J, Jiang X, Zou WB, Wang YC, Zhou W, Li ZS, Liao Z (2021) Use of artificial intelligence for detection of gastric lesions by magnetically controlled capsule endoscopy. Gastrointest Endosc 93:133-139.e134

37. Aoki T, Yamada A, Kato Y, Saito H, Tsuboi A, Nakada A, Niikura R, Fujishiro M, Oka S, Ishihara S, Matsuda T, Nakahori M, Tanaka S, Koike K, Tada T (2020) Automatic detection of blood content in capsule endoscopy images based on a deep convolutional neural network. J Gastroenterol Hepatol 35:1196-1200

38. Yamada A, Niikura R, Otani K, Aoki T, Koike K (2020) Automatic detection of colorectal neoplasia in wireless colon capsule endoscopic images using a deep convolutional neural network. Endoscopy.

39. Otani K, Nakada A, Kurose Y, Niikura R, Yamada A, Aoki T, Nakanishi H, Doyama H, Hasatani K, Sumiyoshi T, Kitsuregawa M, Harada T, Koike K (2020) Automatic detection of different types of small-bowel lesions on capsule endoscopy images using a newly developed deep convolutional neural network. Endoscopy 52:786-791.

40. Caroppo A, Leone A, Siciliano P (2021) Deep transfer learning approaches for bleeding detection in endoscopy images. Comput Med Imaging Graph 88:101852

41. Iddan G, Meron G, Glukhovsky A, Swain P (2000) Wireless capsule endoscopy. Nature 405:417

42. Spada C, Hassan C, Bellini D, Burling D, Cappello G, Carretero C, Dekker E, Eliakim R, de Haan M, Kaminski MF, Koulaouzidis A, Laghi A, Lefere P, Mang T, Milluzzo SM, Morrin M, McNamara D, Neri E, Pecere S, Pioche M, Plumb A, Rondonotti E, Spaander MC, Taylor S, Fernandez-Urien I, van Hooft JE, Stoker J, Regge D (2021) Imaging alternatives to colonoscopy: CT colonography and colon capsule. European Society of Gastrointestinal Endoscopy (ESGE) and European Society of Gastrointestinal and Abdominal Radiology (ESGAR) Guideline-update 2020. Eur Radiol 31:2967-2982 
43. Trasolini R, Byrne MF (2021) Artificial intelligence and deep learning for small bowel capsule endoscopy. Dig Endosc 33:290-297

44. McAlindon ME, Ching HL, Yung D, Sidhu R, Koulaouzidis A (2016) Capsule endoscopy of the small bowel. Ann Transl Med 4:369

45. Giger ML (2018) Machine learning in medical imaging. J Am Coll Radiol 15:512-520

46. Le Berre C, Sandborn WJ, Aridhi S, Devignes MD, Fournier L, Smail-Tabbone M, Danese S, Peyrin-Biroulet L (2020) Application of artificial intelligence to gastroenterology and hepatology. Gastroenterology 158:76-94.e72

47. Mohan BP, Khan SR, Kassab LL, Ponnada S, Chandan S, Ali T, Dulai PS, Adler DG, Kochhar GS (2021) High pooled performance of convolutional neural networks in computer-aided diagnosis of GI ulcers and/or hemorrhage on wireless capsule endoscopy images: a systematic review and meta-analysis. Gastrointest Endosc 93:356-364.e354

48. Soffer S, Klang E, Shimon O, Nachmias N, Eliakim R, Ben-Horin S, Kopylov U, Barash Y (2020) Deep learning for wireless capsule endoscopy: a systematic review and meta-analysis. Gastrointest Endosc 92:831-839.e838

49. Koulaouzidis A, Dabos KJ (2013) Looking forwards: not necessarily the best in capsule endoscopy? Ann Gastroenterol 26:365-367
50. Klang E (2018) Deep learning and medical imaging. J Thorac Dis 10:1325-1328

51. Niv Y (2008) Efficiency of bowel preparation for capsule endoscopy examination: a meta-analysis. World J Gastroenterol 14:1313-1317

52. Noorda R, Nevárez A, Colomer A, Pons Beltrán V, Naranjo V (2020) Automatic evaluation of degree of cleanliness in capsule endoscopy based on a novel CNN architecture. Sci Rep 10:17706

53. Leenhardt R, Souchaud M, Houist G, Le Mouel JP, Saurin JC, Cholet F, Rahmi G, Leandri C, Histace A, Dray X (2020) A neural network-based algorithm for assessing the cleanliness of small bowel during capsule endoscopy. Endoscopy. https://doi.org/10. 1055/a-1301-3841

54. Liao Z, Gao R, Xu C, Li ZS (2010) Indications and detection, completion, and retention rates of small-bowel capsule endoscopy: a systematic review. Gastrointest Endosc 71:280-286

55. Gan T, Liu S, Yang J, Zeng B, Yang L (2020) A pilot trial of Convolution Neural Network for automatic retention-monitoring of capsule endoscopes in the stomach and duodenal bulb. Sci Rep 10:4103

Publisher's Note Springer Nature remains neutral with regard to jurisdictional claims in published maps and institutional affiliations. 